Kansas State University Libraries

New Prairie Press

\title{
WHEN SHOULD RANDOM EFFECTS BE INCLUDED IN ESTIMABLE FUNCTIONS AND WHEN THEY SHOULD NOT?
}

David C. Blouin

Follow this and additional works at: https://newprairiepress.org/agstatconference

Part of the Agriculture Commons, and the Applied Statistics Commons

\section{cc) (1) $\Theta$}

This work is licensed under a Creative Commons Attribution-Noncommercial-No Derivative Works 4.0 License.

\section{Recommended Citation}

Blouin, David C. (1992). "WHEN SHOULD RANDOM EFFECTS BE INCLUDED IN ESTIMABLE FUNCTIONS AND WHEN THEY SHOULD NOT?," Conference on Applied Statistics in Agriculture. https://doi.org/ $10.4148 / 2475-7772.1406$

This is brought to you for free and open access by the Conferences at New Prairie Press. It has been accepted for inclusion in Conference on Applied Statistics in Agriculture by an authorized administrator of New Prairie Press. For more information, please contact cads@k-state.edu. 


\title{
WHEN SHOULD RANDOM EFFECTS BE INCLUDED IN ESTIMABLE FUNCTIONS AND WHEN THEY SHOULD NOT?
}

\author{
David C. Blouin \\ Department of Experimental Statistics \\ Louisiana State University \\ Baton Rouge, Louisiana 70803
}

\begin{abstract}
In the mixed model, the behavior of linear functions of the fixed and random effects is examined. It is found that inclusion of certain functions of random effects can lead to estimators which are equivalent to those under a fixed effects model and are inconsistent with the inherent structure of the mixed model. Three examples are presented which illustrate the behavior of linear functions of the fixed and random effects. These functions represent the broad, narrow and intermediate inference spaces as introduced by McLean, Sanders and Stroup (1991). Which random effects should be included in the model is discussed. Random effects representing experimental error units are candidates for inclusion in estimable functions. Inclusion of experimental unit effects in estimable functions can lead to misleading results.
\end{abstract}

KEY WORDS: mixed model, inference space, predictable functions, estimability, covariance structure, fixed effects structure, shrinkage estimators.

\section{INTRODUCTION}

In this paper, the mixed analysis of variance model is considered with a focus on a particular issue: defining appropriate estimable functions of the fixed and random effects. McLean, Sanders and Stroup (1991) introduced and discussed the distinction between broad, narrow and intermediate inference spaces. These spaces are represented by linear functions of the fixed and random effects with distinctive characteristics. The major goal is to examine these characteristics.

To set the stage, let $\underline{\theta}$ denote the entire $\mathrm{t} \times 1$ vector of fixed effects and let $\underline{\delta}$ denote the entire $p \times 1$ vector of random effects exclusive of $\varepsilon$ as the $N \times 1$ vector of random error terms. The $\mathrm{N} \times 1$ vector of observations $\mathrm{Y}$ can be expressed in the mixed model as

$$
\mathrm{Y}=\mathrm{W} \underline{\theta}+\mathrm{U} \underline{\delta}+\underline{\varepsilon}
$$

where $\mathrm{W}$ and $\mathrm{U}$ are the $\mathrm{N} \times \mathrm{t}$ and $\mathrm{N} \times \mathrm{p}$ fixed effects and random effects design matrices, respectively. For all but the simplest of mixed models, the vector of random effects will 
contain multiple sets of random effects. Let $\underline{\delta}_{r}$ denote the vector of random effects for the $\mathrm{r}^{\text {th }}$ set of random effects and let $\mathrm{s}>1$ denote the number of sets of random effects. The vector of random effects can be partitioned accordingly as

$$
\underline{\delta}=\left[\underline{\delta}_{1}^{\prime}: \underline{\delta}_{2}^{\prime}: \ldots: \underline{\delta}_{r}^{\prime}: \ldots: \underline{\delta}_{s}^{\prime}\right]^{\prime}
$$

so that the mixed model becomes

$$
\mathrm{Y}=\mathrm{W} \underline{\theta}+\sum_{r} \underline{\delta}_{r}+\underline{\varepsilon} .
$$

For example, consider a variety trial experiment involving different plant spacings in a randomized complete block design at each of several locations. The scalar model might be written as

$$
y_{i j k l}=\mu_{k l}+\rho_{i}+\gamma_{i j}+\varepsilon_{i j k l}: \mu_{k l}=\mu+\alpha_{k}+\beta_{l}+\alpha \beta_{k l}
$$

where the vector of fixed effects $\underline{\theta}$ would contain the overall mean $\mu$, the $\alpha_{k}$ variety effects, the $\beta_{l}$ spacing effects and the $\alpha \beta_{k l}$ interaction effects while the vector of random effects $\underline{\delta}$ would contain $\mathrm{s}=2$ sets of random effects: the $\rho_{i}$ location effects in $\underline{\delta}_{1}$ and the $\gamma_{i j}$ block within location effects in $\underline{\delta}_{2}$.

Using Henderson's mixed model equations (Henderson, 1984) under the assumptions that the $\underline{\delta}_{r}$ are multivariate normal with zero mean vector and covariance matrix $\mathrm{G}_{r}^{*}=\phi_{r} \mathrm{I}_{r}$ and $\underline{\varepsilon}$ is multivariate normal with zero mean vector and covariance matrix $\mathrm{R}=\phi_{0} \mathrm{I}_{\mathrm{N}}$, a solution to the mixed equations as given by

$$
\mathrm{P} \Omega=\left[\begin{array}{cc}
\mathrm{W}^{\prime} \mathrm{W} & \mathrm{W}^{\prime} \mathrm{U} \\
\mathrm{U}^{\prime} \mathrm{W} & \mathrm{U}^{\prime} \mathrm{U}+\mathrm{G}^{-1}
\end{array}\right]\left[\begin{array}{l}
\underline{\theta} \\
\underline{\delta}
\end{array}\right]=\mathrm{T}=\left[\begin{array}{c}
\mathrm{W}^{\prime} \mathrm{Y} \\
\mathrm{U}^{\prime} \mathrm{Y}
\end{array}\right]: \mathrm{G}=\text { block diag }_{s}\left[\frac{\phi_{r}}{\phi_{0}} \mathrm{I}_{r}\right]
$$

would yield $\hat{\Omega}=\mathrm{CT}$ with $\mathrm{C}=\mathrm{P}^{-}$. The matrix $\mathrm{C}$ is partitioned according to

$$
\mathrm{C}=\left[\begin{array}{ll}
\mathrm{C}_{f f} & \mathrm{C}_{f r} \\
\mathrm{C}_{r f} & \mathrm{C}_{r r}
\end{array}\right]
$$

The $\hat{\Omega}$ solution vector contains $\underline{\hat{\theta}}$ as BLUE for $\mathrm{H} \theta=\left(\mathrm{W}^{\prime} \mathrm{W}\right)^{-}\left(\mathrm{W}^{\prime} \mathrm{W}\right) \underline{\theta}$ since $\mathrm{W}$ may not be full rank and $\underline{\hat{\delta}}$ as BLUP for the $\underline{\delta}$ random effects vector. The covariance matrix for $\underline{\hat{\theta}}$ and $\underline{\hat{\delta}}-\underline{\delta}$ is $\phi_{0} \mathrm{C}$. Consideration is given to predictable functions of the form

$$
\mathrm{K} \underline{\hat{\theta}}+\mathrm{M}(\underline{\hat{\delta}}-\underline{\delta})=\mathrm{K} \underline{\hat{\theta}}+\sum_{r} \mathrm{M}_{r}\left(\underline{\hat{\delta}}_{r}-\underline{\delta}_{r}\right)
$$


provided $\mathrm{K} \underline{\theta}$ is estimable. Broad, narrow and intermediate inference spaces are distinquished according to the $\mathrm{M}_{r}$ functions. If all $\mathrm{M}_{r}$ are null, then the inference space is said to be broad. If all $\mathrm{M}_{r}$ are not null, then the inference space is said to be narrow. If only some $\mathrm{M}_{r}$ are null, then the inference space is said to be intermediate.

Two questions arise which are the principal focus of this paper. The first is which effects should be included as random effects in a model and the second is which random effects should or should not be candidates for inclusion in a predictable function as defined by $\mathrm{M}_{r}$. The focus of the discussion will be to raise issues that should be addressed in defining meaningful functions of the fixed and random effects in applied mixed models.

\section{SPECIFYING A MIXED MODEL}

In specifying an effects model, all possible sets of combinations of effects are sometimes included. For example, in the randomized complete block design with a two way factorial A x B treatment structure and multiple observations per block by treatment combination, all treatment combinations are assigned completely at random to the experimental units within each block. If blocks are considered to be random, then the experimental units within each block would be intrinsically correlated and the scalar model might be formulated as

$$
y_{i j k l}=\mu+\alpha_{j}+\beta_{k}+\alpha \beta_{j k}+\rho_{i}+\varepsilon_{i j k l}
$$

with observation covariance structure given by

$$
\begin{aligned}
\operatorname{Cov}\left(y_{i j k l}, y_{a b c d}\right) & =\phi_{0}+\phi_{1} & & \text { if } i j k l=a b c d \\
& =\phi_{1} & & \text { if } i=a, j k l \neq b c d \\
& =0 & & \text { if } i \neq a
\end{aligned}
$$

For the model in (2.1), the fixed effect vector $\underline{\theta}$ for the model in (1.3) would contain $\mu$ as the overall mean, the $\alpha_{j}$ as the treatment A main effects, the $\beta_{k}$ as the treatment B main effects and the $\alpha \beta_{j k}$ as the treatment A x B interaction effects while the random effects vector $\underline{\delta}$ for the model in (1.3) would simply contain the $\rho_{i}$ block effects with variance $\phi_{1}$.

The model in (2.1) may be controversial for two reasons. The model does not contain a term for identifying the individual experimental units to which the treatments are assigned and ignores the fact that the observations within each experimental unit are intrinsically correlated. Letting $\rho \alpha \beta_{i j k}$ denote the individual experimental units within each block to which the treatments are assigned, the more appropriate mixed model would become

$$
y_{i j k l}=\mu+\alpha_{j}+\beta_{k}+\alpha \beta_{j k}+\rho_{i}+\rho \alpha \beta_{i j k}+\varepsilon_{i j k l}
$$

with observation covariance structure given by 


$$
\begin{aligned}
\operatorname{Cov}\left(y_{i j k l}, y_{a b c d}\right) & =\phi_{0}+\phi_{1}+\phi_{2} & & \text { if } i j k l=a b c d \\
& =\phi_{1}+\phi_{2} & & \text { if } i j k=a b c, l \neq d \\
& =\phi_{1} & & \text { if } i=a, j k \neq b c \\
& =0 & & \text { if } i \neq a
\end{aligned}
$$

where $\phi_{1}$ is the variance component for the random block effects and $\phi_{2}$ is the variance component for the random experimental unit effects. A corresponding result is that the random effects vector $\underline{\delta}$ for the model in (1.3) would contain the block effects in $\underline{\delta}_{1}$ and the experimental unit effects in $\underline{\delta}_{2}$.

For the model in (2.3) and (2.4), the $\rho \alpha \beta_{i j k}$ term would give rise to the experimental error term for all hypotheses on the fixed treatment effects. To extend the block by treatment combination effects further according to

$$
y_{i j k l}=\mu+\alpha_{j}+\beta_{k}+\alpha \beta_{j k}+\rho_{i}+\rho \alpha_{i j}+\rho \beta_{i k}+\rho \alpha \beta_{i j k}+\varepsilon_{i j k l} \text {, }
$$

the $\rho \alpha_{i j}, \rho \beta_{i k}$ and $\rho \alpha \beta_{i j k}$ effects would give rise to different experimental error terms for the $\alpha_{j}, \beta_{k}$ and $\alpha \beta_{j k}$ fixed effects, respectively. But this model would be suspect since it would require assuming that each block by treatment component effect would provide sources of covariance over and above those already contributed by the block effects and the experimental unit effects. The resulting covariance structure would become

$$
\begin{aligned}
\operatorname{Cov}\left(y_{i j k l}, y_{a b c d}\right) & =\phi_{0}+\phi_{1}+\phi_{2}+\phi_{3}+\phi_{4} & & \text { if } i j k l=a b c d \\
& =\phi_{1}+\phi_{2}+\phi_{3}+\phi_{4} & & \text { if } i j k=a b c, l \neq d \\
& =\phi_{1}+\phi_{3} & & \text { if } i k=a c, j \neq b \\
& =\phi_{1}+\phi_{2} & & \text { if } i j=a b, k \neq c \\
& =\phi_{1} & & \text { if } i=a, j \neq b, k \neq c \\
& =0 & & \text { if } i \neq a .
\end{aligned}
$$

The conclusion here is threefold. Which random effects are to be included in the model determines the observation covariance structure which should be consistent with the nature and behavior of the experimental material and which random effects are to be included in the model automatically become candidates for inclusion in narrow or intermediate inference spaces through $\mathrm{M}_{r}$. However, those effects which represent the experimental error structure are truly random events and should not be included in predictable functions as effects which are repeatable in any real sense. 


\section{SPECIFYING PREDICTABLE FUNCTIONS}

Three examples are presented and discussed. The first example illustrates that narrow inference spaces under certain circumstances are essentially equivalent to treating the model as a pure fixed effects model. The data in (3.3) are those used by McLean, Sanders and Stroup (1991). The experimental problem involved two machines as fixed effects, three operators as random effects and two production trials per cell. The model becomes

$$
y_{i j k l}=\mu+\alpha_{i}+\rho_{j}+\gamma_{i j}+\varepsilon_{i j k}
$$

with $\mathrm{y}_{i j k}$ in (3.3) as the response on trial $k(=1,2)$ by operator $j(=1,2,3)$ on machine $i$ $(=1,2)$, the $\alpha_{i}$ as the fixed machine effects, the $\rho_{j}$ as the random operator effects with variance $\phi_{1}$, the $\gamma_{i j}$ as the random machine by operator cell effects with variance $\phi_{2}$, the $\varepsilon_{i j k}$ as the random error effects with variance $\phi_{0}$ and covariance structure

$$
\begin{aligned}
\operatorname{Cov}\left(y_{i j k l}, y_{a b c d}\right) & =\phi_{0}+\phi_{1}+\phi_{2} & & \text { if } i j k=a b c \\
& =\phi_{1}+\phi_{2} & & \text { if } i j=a b, k \neq c \\
& =\phi_{1} & & \text { if } j=b, i \neq a \\
& =0 & & \text { if } j \neq b .
\end{aligned}
$$

Hence, for the model in (1.3), the $\underline{\theta}$ fixed effects vector contains the $\mu$ and $\alpha_{i}$ effects while the $\underline{\delta}$ random effects vector contains the $\gamma_{i j}$ effects in $\underline{\delta}_{2}$ and the $\rho_{\mathrm{j}}$ effects in $\underline{\delta}_{1}$. For all results that are to be presented, the actual data are contained in the observation vector

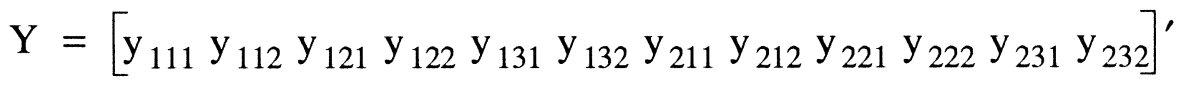

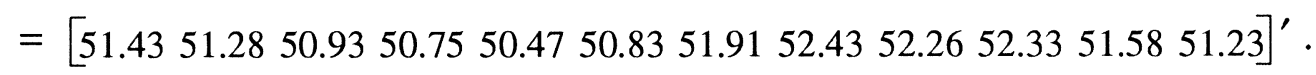

All results were obtained from GLMM, a general linear mixed model program (Blouin and Saxton, 1989; Blouin, Saxton and Koonce, 1989). Using REML (Corbeil and Searle, 1974), the estimators of the variance components for the error, operator, and machine by operator random effects are $0.0485=\operatorname{est}\left(\phi_{0}\right), 0.0510=\operatorname{est}\left(\phi_{1}\right)$ and $0.1073=\operatorname{est}\left(\phi_{2}\right)$, respectively. It is noted that Henderson's Method 3, MIVQUE0 and Henderson's REML using quadratics developed by LaMotte (1971) all yield the same estimators. The fixed effects solution vector for the overall mean and the machine effects becomes

$$
\underline{\hat{\theta}}=[51.96-1.01 \text { null }]^{\prime}=\operatorname{est}\left[\mu+\alpha_{2} \alpha_{1}-\alpha_{2} \text { null }\right]^{\prime}=\operatorname{est}(\mathrm{H} \theta) .
$$


The random effects solution vector for the $\rho_{i}$ operator effects becomes

$$
\hat{\delta}_{1}=\left[\begin{array}{lll}
0.2295 & 0.0851-0.3146
\end{array}\right]^{\prime}
$$

and the random effects solution vector for the $\gamma_{i j}$ operator by machine effects becomes

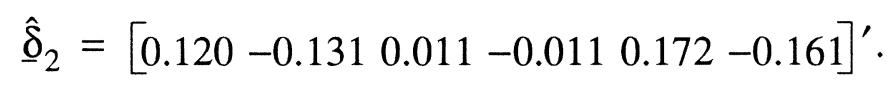

If the broad inference space functions with $\mathrm{M}_{1}$ and $\mathrm{M}_{2}$ both null as defined by

$$
\mathrm{L}=\left[\begin{array}{llllllllllll}
1 & 1 & 0 & 0 & 0 & 0 & 0 & 0 & 0 & 0 & 0 & 0 \\
1 & 0 & 1 & 0 & 0 & 0 & 0 & 0 & 0 & 0 & 0 & 0
\end{array}\right]
$$

are employed, then the $\mathrm{L} \hat{\Omega}$ estimators of the fixed effect machine means would be equal to $\bar{y}_{i . .}=\{50.95,51.96\}$ with standard errors equal to $0.2467=\operatorname{se}\left(\bar{y}_{i . .}\right)$. Conversely, if the narrow inference space functions with neither $\mathrm{M}_{r}$ null as defined by

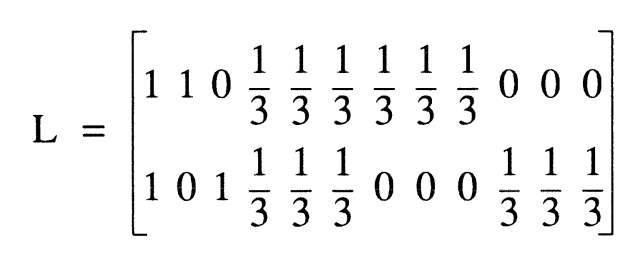

are employed, then the $\mathrm{L} \hat{\Omega}$ estimators of the fixed effect machine means would again be equal to $\overline{\mathrm{y}}_{i . .}=\{50.95,51.96\}$, but with standard errors $0.0899=\operatorname{se}\left(\overline{\mathrm{y}}_{i . .}\right)$. However, these standard errors are equivalent to the standard errors that would be obtained if the model had been defined as a completely fixed effects model. Hence, these standard errors are incorrect and too small since the model is not a fixed effects model. A related issue is that the $\mathrm{M}_{2}$ coefficients are applied to the experimental unit effects. These might be considered to be truly random events as part of the error structure and unlikely candidates for inclusion. Hence, the intermediate inference space functions with

$$
\mathrm{L}=\left[\begin{array}{llllllllllll}
1 & 1 & 0 & \frac{1}{3} & \frac{1}{3} & \frac{1}{3} & 0 & 0 & 0 & 0 & 0 & 0 \\
1 & 0 & 1 & \frac{1}{3} & \frac{1}{3} & \frac{1}{3} & 0 & 0 & 0 & 0 & 0 & 0
\end{array}\right]
$$

might be considered with $0.1584=$ se $\left(\overline{\mathrm{y}}_{i . .}\right)$. Here, the $\rho_{i}$ effects are conceptualized as part of the treatment structure which might appear in other replications of the experiment.

The behavior of these broad, intermediate and narrow inference space functions with respect to standard errors is instructive. By way of introduction, simply consider a randomized block design with a single observation per cell, $\mathrm{t}=2$ treatments (indexed by $i$ ), $\mathrm{r}$ $=3$ blocks (indexed by $j$ ) and data given by $\mathrm{y}_{i j}=\left\{\mathrm{y}_{11}=32, \mathrm{y}_{12}=34, \mathrm{y}_{13}=31, \mathrm{y}_{21}=29\right.$ $\left., y_{22}=31, y_{23}=30\right\}$. The estimators of the variance components for error and blocks are 
$0.66667=\operatorname{est}\left(\phi_{0}\right)$ and $1.00000=\operatorname{est}\left(\phi_{1}\right)$, respectively. The treatment means are $\overline{\mathrm{y}}_{i .}=\{32.333,30.000\}$ with standard errors equal to

$$
\operatorname{se}\left(\bar{y}_{i .}\right)=0.7454=\sqrt{\frac{\hat{\phi}_{0}+\hat{\phi}_{1}}{\mathrm{r}}}
$$

As $\phi_{1}$ increases relative to $\phi_{0}$, the standard error of the treatment means increases. However, consider the narrow inference functions of the fixed and random effects as in

$$
\mathrm{L}=\left[\begin{array}{llllll}
1 & 1 & 0 & \frac{1}{3} & \frac{1}{3} & \frac{1}{3} \\
1 & 0 & 1 & \frac{1}{3} & \frac{1}{3} & \frac{1}{3}
\end{array}\right]
$$

L $\hat{\Omega}$ will again yield the same treatment means, but the standard errors of the treatment means are too small and equivalent to the fixed effects model solution given by

$$
\operatorname{se}\left(\bar{y}_{i .}\right)=0.4714=\sqrt{\frac{\hat{\phi}_{0}}{\mathrm{r}}} .
$$

In contrast, under a fixed effects model, consider the overall mean and the block means given by $\overline{\mathrm{y}}_{. .}=31.16667$ and $\overline{\mathrm{y}}_{. j}=\{30.5,32.5,30.5\}$ with se $\left(\overline{\mathrm{y}}_{. .}\right)=0.33333$ and se $\left(\bar{y}_{. j}\right)=0.57735$ as the corresponding standard errors. In a fixed effects model, the functions that would produce the block means would be

$$
\mathrm{L}=\left[\begin{array}{llllll}
1 & \frac{1}{2} & \frac{1}{2} & 1 & 0 & 0 \\
1 & \frac{1}{2} & \frac{1}{2} & 0 & 1 & 0 \\
1 & \frac{1}{2} & \frac{1}{2} & 0 & 0 & 1
\end{array}\right]
$$

But using these same functions under the mixed model, the corresponding estimators of the mixed model block means and their corresponding standard errors would be equal to $\mathrm{L} \hat{\Omega}=\{30.66667,32.16667,30.66667\}$ and $\mathrm{se}(\mathrm{L} \hat{\Omega})=0.52705$ for all elements in $\mathrm{L} \hat{\Omega}$. Hence, the mixed model block means tend to shrink toward the overall mean with a reduced standard error. Let the ratio of $\phi_{0}$ to $\phi_{1}$ range from zero to infinity. The behavior of the L $\hat{\Omega}$ mixed model block means and their standard errors are graphically depicted in Figure 1 and Figure 2, respectively. These figures show that as the variance component ratio increases, the "best predictor" of the block response approaches the overall mean with a reduced standard error. Alternatively, as the block variance increases, the variance ratio decreases and the individual fixed effect block means are the "best predictors" of the 
the block response. Hence, under the mixed model, as the block variance decreases, the "best predictors" of the block responses approach the overall mean with a reduced standard error.

Returning to the machine by operator problem, let the variance ratios be $\eta_{1}=\phi_{0} / \phi_{1}$ for the $\rho_{i}$ effects and $\eta_{2}=\phi_{0} / \phi_{1}$ for the $\gamma_{i j}$ effects. Consider the fixed machine effect means. The estimators for the broad inference function as in (3.7), the narrow inference function as in (3.8) and the intermediate inference function as in (3.9) will all be equivalent and equal to $\bar{y}_{i . .}=\{50.95,51.96\}$, but the standard errors will differ as a function of the variance ratios. Regardless of the variance ratios, the narrow inference function will yield the fixed effects model results of $0.0899=\operatorname{se}\left(\bar{y}_{i . .}\right)$. Hence, the narrow inference function treats the model as fixed, not mixed. Fix the value of $\phi_{0}$ and let $\eta_{1}$ and $\eta_{2}$ increase, or equivalently, let $\phi_{1}$ and $\phi_{2}$ decrease. For any given $\eta_{1}$ and as $\eta_{2}$ approaches infinity, the standard error of the mean for the intermediate inference space approaches the standard error of the mean for the narrow inference space. Similarly, for any given $\eta_{2}$ and as $\eta_{1}$ approaches infinity, the standard error of the mean for the broad inference space approaches the standard error of the mean for the intermediate inference space. Hence, as both variance ratios increase or both variance components decrease, the mixed model approaches a fixed effects model. The result is that under a mixed model, the standard error of the mean from the narrow inference space is too small.

In contrast, consider functions which focus on the random operators and the random operator by machine effects. For discussion and comparative purposes, the fixed effects model means with standard errors are given by $\bar{y}_{\ldots}=51.425$ and $0.06359=\operatorname{se}\left(\bar{y}_{\ldots}\right)$, $\overline{\mathrm{y}}_{. j .}=\{30.66667,32.16667,30.66667\}$ and $0.11014=\mathrm{se}\left(\overline{\mathrm{y}}_{. j .}\right)$ as the standard error for each mean, and $\bar{y}_{i j .}=\{51.355,50.840,50.650,52.170,52.295,51.405\}$ with standard errors equal to $0.15576=\operatorname{se}\left(\bar{y}_{i j}\right)$. The functions for random operators yield

$$
\mathrm{L}_{\rho} \hat{\Omega}=\left[\begin{array}{llllllllllll}
1 & \frac{1}{2} & \frac{1}{2} & 1 & 0 & 0 & \frac{1}{2} & 0 & 0 & \frac{1}{2} & 0 & 0 \\
1 & \frac{1}{2} & \frac{1}{2} & 0 & 1 & 0 & 0 & \frac{1}{2} & 0 & 0 & \frac{1}{2} & 0 \\
1 & \frac{1}{2} & \frac{1}{2} & 0 & 0 & 1 & 0 & 0 & \frac{1}{2} & 0 & 0 & \frac{1}{2}
\end{array}\right] \hat{\Omega}=\left[\begin{array}{l}
51.73656 \\
51.55788 \\
51.06306
\end{array}\right]
$$

with standard errors equal to $0.107026=\operatorname{se}\left(\mathrm{L}_{\rho} \hat{\Omega}\right)$ while the corresponding functions for the random machine by operator effects yield

$$
\mathrm{L}_{\rho \gamma} \hat{\Omega}=\left[\begin{array}{llllllllllll}
1 & 1 & 0 & 1 & 0 & 0 & 1 & 0 & 0 & 0 & 0 & 0 \\
1 & 1 & 0 & 0 & 1 & 0 & 0 & 1 & 0 & 0 & 0 & 0 \\
1 & 1 & 0 & 0 & 0 & 1 & 0 & 0 & 1 & 0 & 0 & 0 \\
1 & 0 & 1 & 1 & 0 & 0 & 0 & 0 & 0 & 1 & 0 & 0 \\
1 & 0 & 1 & 0 & 1 & 0 & 0 & 0 & 0 & 0 & 1 & 0 \\
1 & 0 & 1 & 0 & 0 & 1 & 0 & 0 & 0 & 0 & 0 & 1
\end{array}\right] \hat{\Omega}=\left[\begin{array}{l}
51.29790 \\
50.90237 \\
50.64473 \\
52.17522 \\
52.21338 \\
51.48139
\end{array}\right]
$$


with standard errors equal to $0.14484=$ se $\left(\mathrm{L}_{\gamma} \hat{\Omega}\right)$. As before, fix the value of $\phi_{0}$ and let $\eta_{1}=\phi_{0} / \phi_{1}$ for the $\rho_{i}$ effects and $\eta_{2}=\phi_{0} / \phi_{1}$ for the $\gamma_{i j}$ effects range from zero to infinity. Some results are that for any $\eta_{1}$ and as $\eta_{2}$ approaches zero, the operator functions in (3.14) yield the fixed effects operator results and the machine by operator functions in (3.15) yield the fixed effects machine by operator results. Hence, as the individual variance components increase separately, the mixed model results for the separate random effects approach the fixed model results. Conversely, as both variance ratios increase to infinity, the operator functions in (3.14) yield the overall mean results under a fixed effects model while the machine by operator functions in (3.15) yield the machine mean results under a fixed effects model. Hence, as both variance components decrease, there is shrinkage to the overall mean for the operator effects and shrinkage to the respective machine means for the machine by operator effects. In all cases, the standard errors under the mixed effects model are smaller than the standard errors under the fixed effects model.

The second example presents a problem with missing cells. The data came from a cotton efficacy trial on the effects of different herbicides at different rates on weed control. The model was $\mathrm{y}_{i j}=\mu_{j}+\rho_{i}+\varepsilon_{i j}$ with a total of twenty treatments in three blocks. The dependent measure was the degree of weed control with zeros possible. Hence, the minimum $\mu_{j}$ is zero. Zero means did exist in the observed data and there was a single missing cell. In particular, observation $\mathrm{y}_{3,17}$ was missing with the data for each block equal to

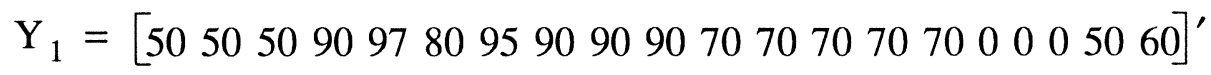

$$
\begin{aligned}
& \mathrm{Y}_{2}=\left[\begin{array}{llllllllllllllllllll}
0 & 80 & 50 & 20 & 90 & 0 & 30 & 80 & 90 & 90 & 90 & 90 & 97 & 99 & 90 & 20 & 0 & 0 & 0 & 0
\end{array}\right]^{\prime}
\end{aligned}
$$

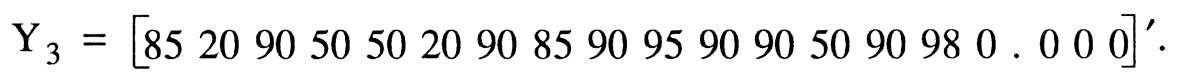

Using Henderson's Method 3, the variance components are $577.74049=\operatorname{est}\left(\phi_{0}\right)$ and $18.5501=\operatorname{est}\left(\phi_{1}\right)$ while the random effects solution vector is

$$
\hat{\delta}=[2.9727-2.2282-0.7445]^{\prime} .
$$

The fixed effects solution $-0.37225=\operatorname{est}\left(\theta_{17}\right)$ for treatment $\mathrm{j}=17$ is precisely equal to

$$
\hat{\theta}_{17}=\bar{y}_{17}-\frac{\hat{\rho}_{1}+\hat{\rho}_{2}}{2}
$$

with standard error se $=17.2329$. Consideration could be given to adding back the function of the random effects as a pseudonarrow inference function which effectively zeros out the random effects in (3.18). The function would be

$$
\mathrm{L}=\left[\begin{array}{llllllllllllllllllllll}
0 & 0 & 0 & 0 & 0 & 0 & 0 & 0 & 0 & 0 & 0 & 0 & 0 & 0 & 0 & 0 & 1 & 0 & 0 & 0 & \frac{1}{2} & \frac{1}{2}
\end{array}\right] \text {, }
$$


but the results of $\mathrm{L} \hat{\Omega}=0$ with standard error $\mathrm{se}=16.9962$ would be precisely equivalent to the results if the model had been executed as a completely fixed effects model.

For a third and final example, rice variety trials in a randomized complete block design with four blocks were conducted at each of three locations. Locations differed in soil types and there were ten lines of rice. Nitrogen treatments were randomly assigned to plots within a block and all lines were randomly assigned to subplots within each plot. Hence, the model becomes

$$
\mathrm{y}_{i j k l}=\mu+\alpha_{i}+\delta_{i j}+\beta_{k}+\alpha \beta_{i k}+\gamma_{i j k}+v_{l}+\alpha v_{i l}+\beta v_{k l}+\alpha \beta v_{i k l}+\varepsilon_{i j k l}
$$

with $\mu$ as the overall mean, the $\alpha_{i}$ as the location effects, the $\delta_{i j}$ as the random block within location experimental error effects for locations, the $\beta_{k}$ as the fixed nitrogen effects, the $\alpha \beta_{i k}$ as the location by nitrogen combination effects, the $\gamma_{i j k}$ as the random nitrogen by block within location experimental error effects for nitrogen and location by nitrogen treatment effects, the $v_{l}$ as the varietal line effects, and the $\alpha v_{i l}$ and the $\beta v_{k l}$ and the $\alpha \beta v_{i k l}$ as the associated factorial effects of line with location and nitrogen treatment effects.

Consider treating the location and varietal line effects as random. All of the crossclassified effects involving the $v_{l}$ line effects could be conceptualized as various environmental by genotypic interaction effects, the $\alpha \beta_{i k}$ could be conceptualized as various environmental by treatment interaction effects, and all other terms could be conceptualized as random expressions of experimental error. Hence, the only fixed effect terms are represented by $\mu$ and the $\beta_{k}$. However, the $\alpha_{i}$ location effects and the $v_{l}$ line effects could be considered arguably as fixed effects. Consider defining functions of the $\alpha_{i}$ location effects (fixed or random), the $\beta_{k}$ soil treatment effects (fixed) or the $v_{l}$ line effects (fixed or random). Let $M_{\alpha}, M_{\delta}, M_{\alpha \beta}, M_{\gamma}, M_{\nu}$ and so forth denote the corresponding partitions of the narrow inference space function. All $\mathrm{M}$ matrices, except $\mathrm{M}_{\delta}$ and $\mathrm{M}_{\gamma}$ as expresssions of functions of random experimental error effect, could be viewed as expresssions of functions of repeatable main effects and interaction effects and would be candidates for inclusion in L functions. $\mathrm{M}_{\delta}$ and $\mathrm{M}_{\gamma}$ as expresssions of functions of experimental error effects would not be candidates for inclusion in narrow or intermediate inference functions. Similarly, all effects except the $\delta_{i j}$ and the $\gamma_{i j k}$ could be classified reasonably as contributing to the treatment structure as well as the observation covariance structure. As such, M matrices corresponding to these effects could be considered for inclusion in defining functions.

\section{SUMMARY}

Although statistical software packages may provide opportunities to examine a rich variety of functions of the fixed and random effects, there may be certain classes of functions which are almost always, if not always, inappropriate. Consideration might be given, as far as is practicable, to increasing the difficulty of gaining access to these functions. As 
has been illustrated and as a negative aspect of using narrow inference functions, the narrow inference functions that were examined lead to results that are equivalent to the results from a completely fixed effects model solution although the specified model is mixed.

\section{REFERENCES}

Blouin, D.C., and Saxton, A.M. (1989). Integrating Fixed Effects Hypothesis Testing with Variance Component Estimation: A Prototype of a Mixed Model Program. Proceedings of the 21st Symposium on the Interface, American Statistical Association, 455-463.

Blouin, D.C., Saxton, A.M., and Koonce, K.L. (1989). A Competely Randomized Design with Missing Cells and Repeated Measures in Time. In Applications of Mixed Models in Agriculture and Related Disciplines, Southern Cooperative Series Bulletin No. 343, 80-103, Louisiana Agricultural Experiment Station, Baton Rouge.

Corbeil, R.R., and Searle, S.R. (1976). Restricted Maximum Likelihood (REML) Estimation of Variance Components in the Mixed Model. Technometrics, 18, 31-38.

Henderson, C.R. (1984). Applications of Linear Models in Animal Breeding. University of Quelp, Quelp, Ontario, Canada.

LaMotte, L.R. (1971). Locally Best Quadratic Estimators of Variance Components. Technical Report 22, University of Kentucky.

McLean, R.A., Sanders, W.L., and Stroup, W.W. (1991). A Unified Approach to Mixed Linear Models. The American Statistician, 45, 54-64. 
Figure 1. Block means as the variance ratio $\phi_{0} / \phi_{1}$ increases.

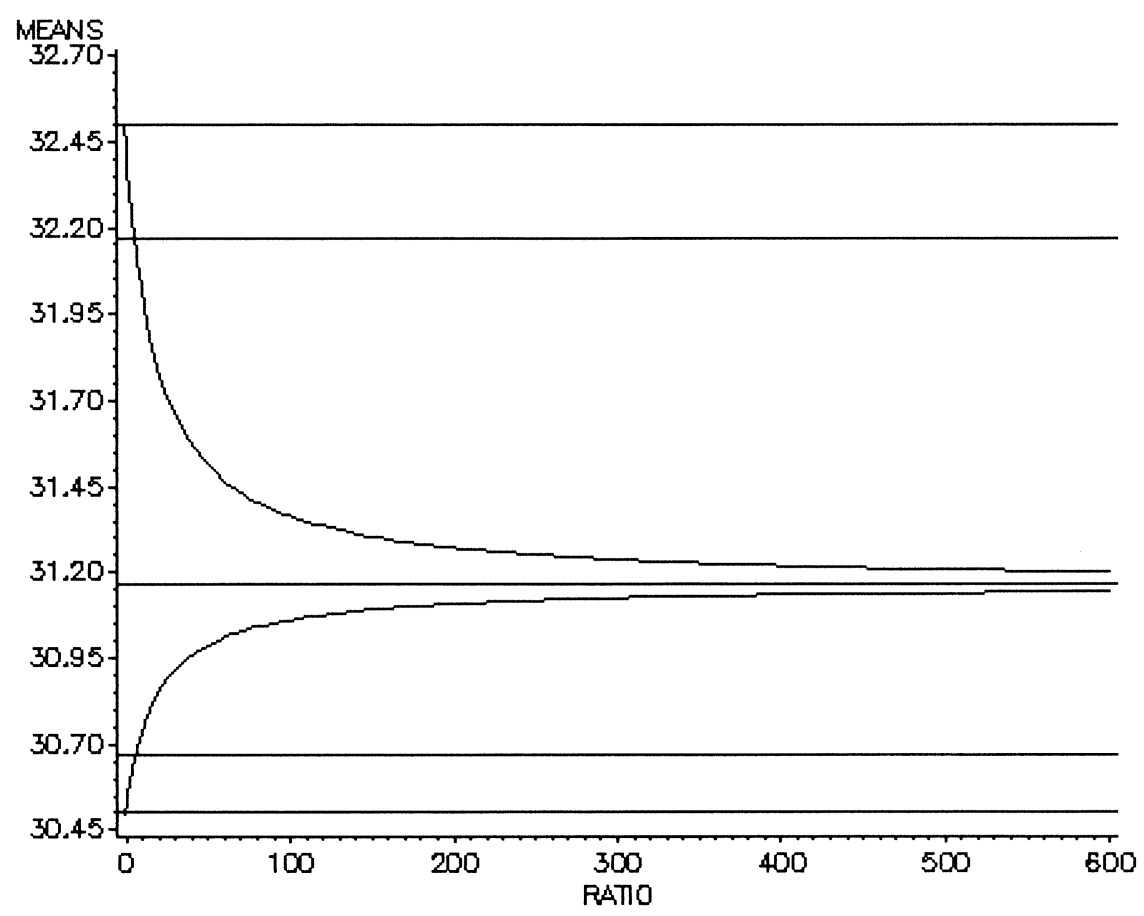

Figure 2. Standard error of block means as the variance ratio $\phi_{0} / \phi_{1}$ increases.

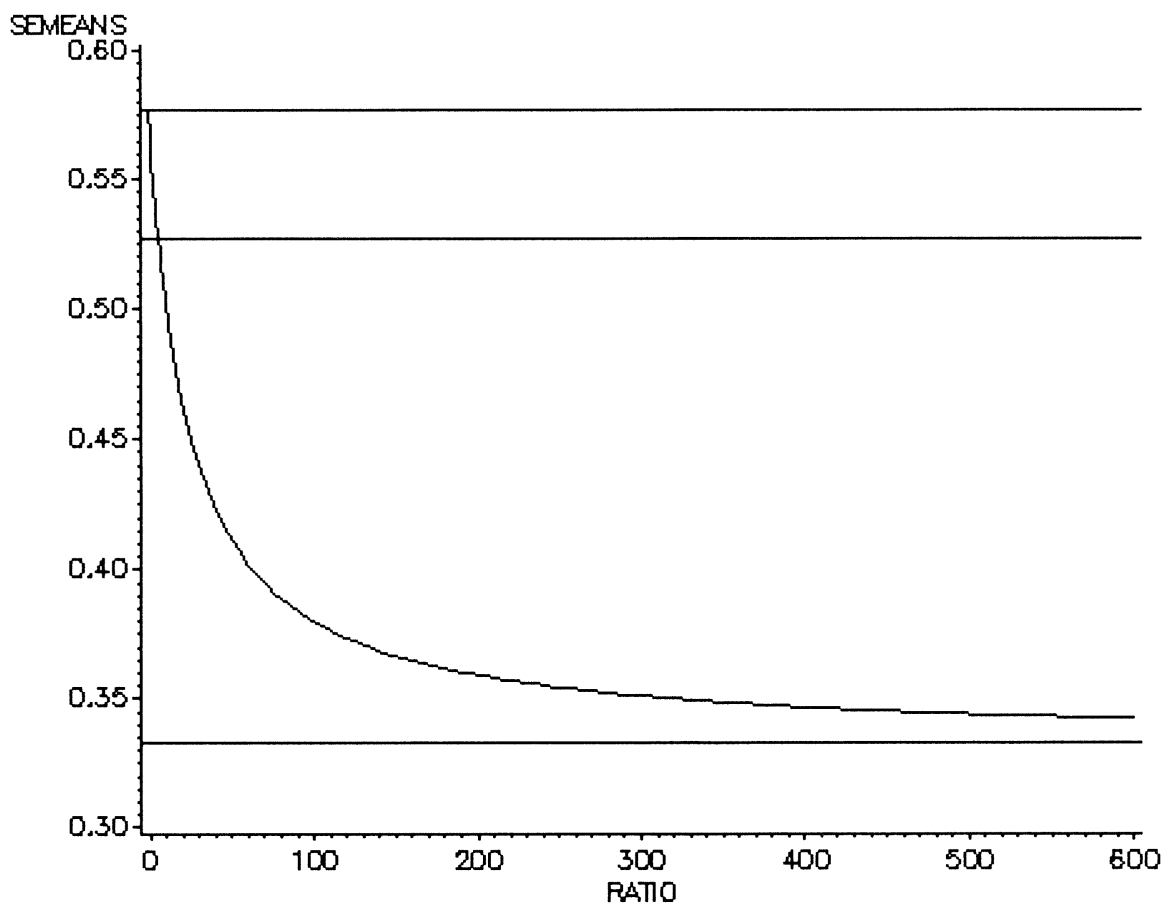

